\title{
CONCEITOS CLÁSSICOS E ABORDAGENS CORRENTES EM INTELIGÊNCIA COMPETITIVA: UM ESTUDO BIBLIOMÉTRICO DE 2005 A 2015
}

\section{RESUMO}

Este artigo identificar os conceitos da Inteligência Competitiva conforme a abordagem dos principais autores referenciados visando a mensuração da evolução do tema, por meio da caracterização das correntes conceituais e funcionais. Para tanto, realizou-se uma pesquisa bibliométrica, em IC, nos anos de 2005 a 2015, período em que a IC começa a se firmar como principal provedora de informação nas organizações. Os dados foram coletados nos periódicos científicos da base internacional Web of Science. Os artigos compilados foram processados por meio do Software Bibexcel, gerando uma matriz de citações e co-citações, que processada, resultou na análise fatorial exploratória, permitindo relacionar os principais autores clássicos ou seminais no tema, bem como as principais correntes que os mesmos defendem. Apresenta-se como principal conclusão que todas as abordagens das correntes identificadas, seja no marketing, na gestão do conhecimento, nos negócios, na tecnologia entre outras, permitem às organizações uma melhor estruturação estratégica. Foi possível identificar também os autores atuais que continuam publicando, com base em suas pesquisas, sobre os temas clássicos bem como as evoluções das abordagens conceituais. Quanto à contribuição teórica destaca-se que de fato, há pouca pesquisa sobre os fundamentos teóricos da inteligência competitiva, deixando a descoberto áreas importantes, como maturidade de uso da inteligência em organizações e teoria dos processos de inteligência.

Palavras-chave: Inteligência Competitiva; Análise de Citações; Correntes Conceituais; Abordagem Evolutiva.

\section{CLASSICAL CONCEPTS AND CURRENT APPROACHES IN COMPETITIVE INTELLIGENCE: A BIBLIOMETRIC STUDY FROM 2005 TO 2015}

\begin{abstract}
This article aims to identify the concepts of Competitive Intelligence according to the approach of the main authors referenced to characterize their conceptual currents and their research areas. In order to do so, a bibliometric study was carried out in IC, from 2005 to 2015, during which time the CI began to establish itself as the main information provider in organizations. The data were collected in the international journals of the Web of Science. The compiled articles were processed through the Bibexcel Software, generating a matrix of citations and co-citations, which processed, resulted in exploratory factorial analysis, allowing to relate the main classical or seminal authors in the theme, as well as the main currents that they defend. It is the main conclusion that all the approaches of the identified chains, be it in marketing, knowledge management, business, technology, among others, allow organizations a better strategic structuring. It was also possible to identify the current authors who continue to publish, based on their research, on the classic themes as well as the evolutions of the conceptual approaches. As for the theoretical contribution, it is emphasized that in fact, there is little research on the theoretical foundations of competitive intelligence, revealing important areas such as maturity of use of intelligence in organizations and theory of intelligence processes.
\end{abstract}

Keywords: Competitive Intelligence; Analysis of Citations; Conceptual Chains; Evolutionary Approach. 


\title{
CONCEPTOS CLÁSICOS Y ENFOQUES CORRIENTES EN INTELIGENCIA COMPETITIVA: UN ESTUDIO BIBLIOMÉTRICO DE 2005 A 2015
}

\section{RESUMEN}

Este artículo identifica los conceptos de la Inteligencia Competitiva conforme el abordaje de los principales autores referenciados visando la medición de la evolución del tema, por medio de la caracterización de las corrientes conceptuales y funcionales. Para ello, se realizó una investigación bibliométrica, en IC, en los años 2005 a 2015, período en que la IC comienza a firmar como principal proveedora de información en las organizaciones. Los datos fueron recogidos en los periódicos científicos de la base internacional Web of Science. Los artículos compilados fueron procesados a través del software Bibexcel, generando una matriz de citas y co-citaciones, que procesada, resultó en el análisis factorial exploratorio, permitiendo relacionar a los principales autores clásicos o seminales en el tema, así como las principales corrientes que los mismos defienden. Se presenta como principal conclusión que todos los enfoques de las corrientes identificadas, sea en el marketing, en la gestión del conocimiento, en los negocios, en la tecnología entre otras, permiten a las organizaciones una mejor estructuración estratégica. Fue posible identificar también a los autores actuales que continúan publicando, sobre la base de sus investigaciones, sobre los temas clásicos así como las evoluciones de los enfoques conceptuales. En cuanto a la contribución teórica se destaca que de hecho, hay poca investigación sobre los fundamentos teóricos de la inteligencia competitiva, dejando a descubierto áreas importantes, como madurez de uso de la inteligencia en organizaciones y teoría de los procesos de inteligencia.

Palabras clave: Inteligencia Competitiva; Análisis de Citas; Corrientes Conceptuales; Enfoque Evolutivo.

\author{
Clébia Ciupak ${ }^{1}$ \\ Leonel Cezar Rodrigues ${ }^{2}$
}

\footnotetext{
${ }^{1}$ Doutora em Administração pela Universidade Nove de Julho - UNINOVE. Professora da Universidade Federal de Mato Grosso - UFMT. Brasil. E-mail: clebia.ciupak@gmail.com

${ }^{2}$ Doutor em Administração Universitária pela Vanderbilt University, Estados Unidos. Professor do Programa de pósGraduação em Administração da Universidade Nove de Julho - PPGA/UNINOVE. Brasil. E-mail: leonel@ uni9.pro.br
} 


\section{INTRODUÇÃO}

Por sua função e natureza, a Inteligência Competitiva (IC) vem assumindo o espaço deixado pelas restrições naturais dos sistemas de informação (SI), como meio de suprir informações a executivos e gerentes nas organizações. Seus objetos, porém informação estruturada e não-estruturada - diferem do objeto dos SI - informações estruturadas e isso tem gerado confusões conceituais e funcionais para a IC. Dessa forma, as constantes mudanças nos cenários político, econômico, tecnológico e social, faz com que as organizações precisem a cada dia, de informações mais completas de seus ambientes de operação. A rápida adaptação às pressões ambientais é vital para a otimização dos processos transacionais, usando recursos de forma otimizada. Manter vantagem competitiva é, dia a dia mais difícil, exigindo das organizações um volume e diversidade maior de informações, de forma a permitir-lhes orientar seus negócios com mais eficiência.

Nesse contexto, a Inteligência Competitiva, como instrumento de apoio administrativo, exerce um papel crucial capaz de "informar, avaliar e predizer acerca de ações ou momentos que possam ter extrema importância para o presente e o futuro" (Rodrigues \& Ricardi, 2007, p. 38). Segundo os autores, "a IC desempenha um papel determinante se for utilizada como um sistema" destinado a apoiar a tomada de decisão, formular estratégias competitivas e integrar as funções organizacionais aos objetivos estratégicos.

Por suas características funcionais mais pragmáticas e utilitaristas, entende-se que a Inteligência Competitiva tenha uma abordagem de forma mais positivista, tanto no mundo empresarial como no meio acadêmico. Por isso, parece haver preocupações da academia quando se trata de colimação de conceitos e teoria básica sobre a IC, em especial pela pouca pesquisa científica que há sobre a mesma (Jaworski, 2004). Jaworski insiste que "a ausência deste conhecimento torna difícil identificar formas de melhorar o processo de geração de Inteligência Competitiva." (Jaworski, 2004, p. 279).

Pode-se deduzir que não se trata apenas de identificar formas alternativas de melhorar o processo da IC, mas principalmente, de se dominar a teoria de seus processos e a amplitude de sua aplicabilidade. Se admitirmos que a informação é um bem estratégico para o sustento da capacidade competitiva, porque descentralizar os processos de prospecção, análise e produção da IC, por exemplo? Quais os fundamentos teóricos que permeiam os processos prospectivos originados na interpretação das necessidades de informação? Essas são apenas algumas de muitas perguntas que aguardam mais pesquisas para serem elucidadas e afinal, contribuírem de forma mais efetiva para o que Jaworski anuncia como "melhorar o processo de geração da IC".

Reflexões dessa natureza sustentaram a motivação para a realização desse artigo, cujos objetivos são, bibliometricamente, identificar os conceitos da Inteligência Competitiva mais usados pelos principais autores neste tema para mensurar-se a evolução do mesmo, visando caracterizar as correntes conceituais e funcionais. $\mathrm{O}$ artigo está estruturado de forma a apresentar primeiramente a introdução, em seguida os antecedentes teóricos sobre a Inteligência Competitiva, depois apresenta-se a metodologia adotada que foi um estudo bibliométrico. Na sequência, apresentou-se os resultados onde, por meio do processamento estatístico, obteve-se a relação das obras mais referenciadas permitindo assim uma análise de citações e também uma análise temática do conteúdo dos resumos dos artigos internacionais obtidos na base Web os Science. Por último tem-se as considerações finais e as referências utilizadas.

\section{REFERENCIAL TEÓRICO}

A seguir apresenta-se os aspectos teóricos e conceituais da Inteligência Competitiva.

\subsection{Inteligência Competitiva}

É essencial que, dada a sua centralidade nos processos decisórios empresariais, a inteligência competitiva seja, a priori, abordada em seus aspectos conceituais e funcionais. Sua importância para os negócios estende-se para além dos elementos internos, uma vez que ela é capaz de direcionar ações estratégicas no entorno das organizações. O estudo bibliométrico realizado para a elaboração desse artigo permitiu um maior direcionamento para a construção desse referencial.

Conceitualmente, a Inteligência Competitiva (IC) é entendida como sendo a arte de localizar, coletar, processar, analisar e disseminar informações a fim de torná-las disponíveis para os usuários (tomadores de decisão). Pelo foco da prospecção, a inteligência competitiva mantém, normalmente, um olhar externo à empresa ou organização. Assim, concentra-se em identificar ameaças e oportunidades correntes e vindouras, que possibilitam antecipar ações de preservação do negócio. Seus processos, porém, respeitam sempre os aspectos éticos e legais (Comai, A., Tena, J., \& Vergara, J.C., 2006). Segundo Heinrichs e Lim (2005), a IC atenta, direta ou indiretamente, para as capacidades necessárias ao apoio às decisões, cujos recursos podem ser os elementos 
necessários para criar, manipular e sintetizar as informações. No mesmo enfoque, Bose (2008) contextualiza a IC como um processo de monitoramento do ambiente competitivo, objetivando fornecer inteligência acionável (informações que sejam utilizáveis no negócio da organização) que irá proporcionar uma vantagem competitiva para a organização. Autores considerados clássicos apontam como um dos objetivos da IC o fornecimento de inteligência acionável, ou seja, informações sintetizadas, processadas e distribuídas, de maneira a suportar o processo gerencial de uma organização (Saayman et al., 2008).

Gainor \& Bouthillier (2014) apontam que a literatura de vários campos de inteligência, como inteligência competitiva e inteligência de negócios, contém críticas sobre as práticas e atividades de medição para determinar o valor da inteligência. Estas críticas incluem preocupações sobre a falta de coerência conceitual, a multiplicidade de abordagens de medição e falta de consenso sobre as melhores práticas, e da falta de evidências de pesquisa para apoiar as atividades de medição, tanto conceitualmente quanto metodologicamente. Para esses autores a inteligência competitiva que pode ser definida como a produção e análise de informações sobre o ambiente competitivo, resultando em produtos de inteligência para uso na tomada de decisão organizacional intenso, é um dos vários campos de inteligência.

Dishman \& Calof (2008) apresentam o conceito de inteligência por meio de um contexto histórico, conforme apresentado no Quadro 1.

\begin{tabular}{|c|c|}
\hline Autores/Ano & Conceito de Inteligência Competitiva \\
\hline $\begin{array}{l}\text { Guyton, 1962; Montgomery e Urbano, 1970; } \\
\text { Pearce, 1976; Montgomery e Weinberg, 1979; } \\
\text { Porter, 1980 }\end{array}$ & $\begin{array}{l}\text { Inteligência como parte da estratégia de marketing, tem } \\
\text { sido proposto como um esforço para aumentar a } \\
\text { competitividade da empresa e seu processo de } \\
\text { planejamento estratégico. }\end{array}$ \\
\hline Fair, 1966 & $\begin{array}{l}\text { Inteligência é uma "Agência Central de Inteligência } \\
\text { Corporativa" cuja função é a de coletar, reunir, organizar, } \\
\text { registrar, recuperar e divulgar informações. }\end{array}$ \\
\hline Kahaner, 1996 & $\begin{array}{l}\text { Inteligência } \begin{array}{c}\text { como } \\
\text { responsáveis pela }\end{array} \text { coleta informações, análise e } \\
\text { divulgação. }\end{array}$ \\
\hline $\begin{array}{l}\text { Aguilar, 1967; Fahey e King, 1977; Fahey et al., } \\
\text { 1982; Hambrick, 1982; Sashittal e Jassawalla, } \\
\text { 2001; Saxby et al., 2002 }\end{array}$ & $\begin{array}{l}\text { Inteligência como um processo de prospecção e } \\
\text { distribuição de informações (Teoria da inteligência) }\end{array}$ \\
\hline $\begin{array}{l}\text { Cleland e King, 1975; Benjamin, 1979; Pearce, } \\
1976\end{array}$ & $\begin{array}{l}\text { uma função do negócio: Inteligência de } \\
\text { s Intelligence - BI). }\end{array}$ \\
\hline Ghoshal e Westney, 1991; Rothschild, 1979 & Inteligência como a Análise da concorrência. \\
\hline $\begin{array}{l}\text { Albagli et al., 1996, Brockhoff, } 1991 \\
\text { Chonko et al., 1991; Guyton, 1962; Maltz e } \\
\text { Kohli, } 1996\end{array}$ & $\begin{array}{l}\text { Inteligência como a prospecção de bases técnicas que } \\
\text { ampliem as bases competitivas da organização. } \\
\text { Inteligência como a prospecção de informações do } \\
\text { comportamento do mercado. }\end{array}$ \\
\hline Shoemaker, 2006 & Inteligência como a visão periférica da organização. \\
\hline Davenport e Harris, 2006 & Inteligência como Análise competitiva. \\
\hline
\end{tabular}

Quadro 1 - Conceitos de Inteligência Competitiva

Fonte: Construído a partir de Dishman, P. L., \& Calof, J. L. (2008). Competitive intelligence: a multiphasic precedent to marketing strategy. European Journal of Marketing, 42(7/8), 766-785.

No Quadro 1 chama a atenção o fato de o conceito de inteligência competitiva ser inicialmente usado como estratégia de marketing. Apesar de sua função preliminar ser de dar suporte ao planejamento estratégico, é compreensível que tenha sido, no começo, utilizada para fortalecer as estratégias de comunicação e penetração mercadológica, já que o marketing estava sendo construído na gestão empresarial à época dos primeiros usos da inteligência.

Parece ser natural que os primeiros movimentos da inteligência tenha nascido na área do marketing e planejamento estratégico (mesmo que este tenha se orientado para a estratégia de marketing). Informações do entorno competitivo, do perfil do mercado e da concorrência são potencialmente importantes para a formulação de estratégia e determinação de metas. Le Bon e Merunka (2006) confirmam esse raciocínio ao apontar que toda a informação recolhida tem o potencial de ser significativa e que os gestores devem considerar a velocidade e frequência da transmissão de informações de mercado, assim como a sua associação com outras que os gestores de informação já possuem, a fim de sustentar os esforços de inteligência. $\mathrm{Na}$ análise dos 
resultados ao longo deste artigo, encontram-se novos constructos do tema bem como o reforço de alguns dos citados no referido Quadro.

Sierra e Rodrigues (2011) apresentam um conceito extraído da Sociedade de Profissionais de Inteligência Competitiva, para os quais a IC consiste em um programa sistemático e ético para reunir, analisar e administrar informações externas que podem afetar planos, decisões e operações de uma empresa. Os autores chamam a atenção para o fato de que a obtenção de informações de modo sistêmico e aplicado aos interesses específicos e estratégicos da organização dizem respeito à inteligência competitiva. Esta foi trazida para o âmbito da Administração, como uma evolução funcional das atividades dos Sistemas de Informação (Gomes e Braga, 2006; apud Sierra e Rodrigues, 2011). Como campo de estudo, porém, a Inteligência Competitiva é uma área ainda pouco explorada.

Neste sentido, Fleisher (2004) já ponderava que há restritas ofertas de cursos e programas e que poucas escolas de negócios ou de gestão têm disciplinas de Inteligência Competitiva. Outro fator que contribui para a baixa evolução conceitual do tema é a escassez de pesquisas. O autor afirma que o campo não tem um conjunto de conhecimentos acordado, textos fundamentais e estudos de caso publicados, fatos esses necessários para profissionalizar um campo. Há ainda ambiguidade sobre onde colocar a Inteligência Competitiva se no negócio, na gestão, ciência da informação, sistemas de informação, jornalismo, ou até mesmo estudos militares. Mesmo dentro de um programa de negócios ou de gestão, existem dúvidas quanto ao fato da Inteligência Competitiva estar atrelada ao marketing, sistemas de informação de gestão, ou à política de negócios /estratégia da organização. Outra abordagem são as tendências econômicas, pois a IC parecia estar decolando na década de 1990, como representado por um grande crescimento no número de membros da Sociedade de Profissionais de Inteligência Competitiva (SCIP), mas depois diminuiu. Estudos anteriores demonstraram declínios na área durante os períodos de recessão, quando as organizações reduziram o número de efetivos de que eles consideraram funções menos essenciais (Fleisher, 2004).

De acordo com Xu, Liao, Li, \& Song (2011), a inteligência competitiva envolve também a identificação precoce de potenciais riscos e oportunidades através da identificação e análise de informações sobre o ambiente de operação de uma organização, para apoiar os gestores na tomada de decisões estratégicas. No mesmo sentido, He, Zha, \& Li (2013) afirmam que a inteligência competitiva pode ajudar as organizações a perceber pontos fortes e fracos, otimizar o negócio, e melhorar a satisfação do cliente. Esses autores apontam ainda que a inteligência competitiva é definida como "a arte de definir, coletar e analisar as informações sobre os produtos concorrentes, promoções, vendas e outras, a partir de fontes externas". O sucesso de uma organização depende de sua capacidade de processar todas as informações julgadas de seu interesse, identificar os fatos históricos que tangenciam sua trajetória e assim poder melhor planejar seu futuro. Nesta mesma linha, Kono, Silva Jr, \& Rodrigues (2014) sinalizam que a IC permite a antecipação de mudanças no meio empresarial; a descoberta de concorrentes novos; a antecipação das ações dos concorrentes e o conhecimento das mudanças que possam afetar os negócios de uma empresa.

Adentrando com mais detalhes a teoria da inteligência competitiva, Rodrigues, Rechziegel, Esteves, \& Pereira Fernandes (2012) defendem que a IC é um processo que envolve atividades de identificação, coleta, desenvolvimento, análise e disseminação da informação, como:

$\checkmark$ Conhecer os concorrentes, mercados, clientes, fornecedores ou outras variáveis do ambiente a ser monitorado.

$\checkmark$ Identificar possíveis fontes de informação.

$\checkmark$ Avaliar a validade, a confiabilidade e a utilidade das informações coletadas.

$\checkmark \quad$ Reunir as informações coletadas de diferentes fontes e integrá-las.

$\checkmark$ Interpretar e analisar as informações de importância estratégica ou tática.

$\checkmark$ Tirar conclusões e recomendar ações.

$\checkmark$ Disseminar e apresentar os resultados analisados para a administração.

$\checkmark$ Fornecer informações complementares de suporte à decisão

A maioria dos autores, porém, trata a inteligência competitiva sob seus aspectos meramente funcionais ou instrumentais, isto é, olham a inteligência como uma mera ferramenta executiva. Seu uso atual nas organizações realmente leva crer que a inteligência seja apenas uma ferramenta gerencial. Contudo, quando se percebe que a administração deixa de ser, paulatinamente, intuitiva para tornar-se gradativamente mais científica, à medida que passa a usar sistemicamente a informação em seus processos decisórios e de formulação de estratégias (Davis \& Stephenson, 2006), começa-se a questionar o mero instrumentalismo da inteligência competitiva. Inteligência mais e mais passa a ter um papel pivotal para a condução dos negócios, quer para a formulação das estratégias competitivas, quer para a construção do adequado modelo de negócio.

\section{METODOLOGIA}

O estudo bibliométrico pode ser caracterizado pela aplicação de métodos estatísticos e matemáticos para avaliar a dinâmica e evolução da informação 
científica e tecnológica. Guedes e Borschiver (2005) caracterizam a bibliometria como sendo uma pesquisa ao longo de publicações de bases científicas e tecnológicas para um período de tempo determinado, numa área específica do conhecimento. Tal metodologia foi utilizada para atender o objetivo deste artigo, que é identificar os conceitos da Inteligência Competitiva conforme a abordagem dos principais autores referenciados visando a mensuração da evolução do tema, por meio da caracterização das correntes conceituais e funcionais. Para este trabalho foi utilizada a base de dados de periódicos internacionais da Web of Science, por ser considerada uma das mais completas, fato que foi constatado pelos pesquisadores ao consultar outras bases que possuíam praticamente os mesmos periódicos. Selecionou-se como palavra-chave "competitive intelligence" e o período compreendido foram os anos de 2005 a 2015 (inclusive).

A busca inicial na referida base trouxe à tona 401 registros totais. Aplicou-se um refinamento na pesquisa na intenção de selecionar apenas "Article" e "Review". Deste refinamento restaram 125 publicações que poderiam ser processados por meio do Software Bibexcel. Observou-se, então, todos os passos constantes do tutorial de Pesquisa já usado em várias bibliometrias [WoS $\rightarrow$.txt $\rightarrow$.doc $\rightarrow$.out $\rightarrow$.1st $\rightarrow$.low $\rightarrow$.cit $\rightarrow$.coc $\rightarrow$.ccc $\rightarrow$.ma2 $\rightarrow$ xlsx $\rightarrow$ SPSS] e obteve-se como resultado, uma matriz de citações e cocitações (analisa a frequência com que um par de referências é citado por outros autores) dos principais autores (Guerrazzi, Brandão, Campos Júnior, \& Lourenço, 2015).

Posteriormente, após o processamento dos dados no Software Estatístico SPSS, foi realizada a Análise Fatorial Exploratória, que permitiu a extração de 7 fatores. As variáveis foram os autores mais citados nos artigos selecionados. Em seguida, dos 125 artigos, baixaram-se 110 para a análise temática, pois 8 não eram da área e foram excluídos e 7 não estavam disponíveis gratuitamente.

\section{DISCUSSÃO E ANÁLISE DOS RESULTADOS}

Após a identificação de cada obra dos autores que fazem parte da AFE procedeu-se aos fatores de agrupamento conforme a corrente de pesquisa abordada por cada um. Listou-se os resumos das 38 obras. A maioria são artigos, mas alguns são livros, denominando-se essa análise como análise de citações, na qual foi possível identificar os autores clássicos ou seminais mais referenciados no período e suas correntes conceituais. Posteriormente, realizou-se uma análise temática de cada artigo utilizado para relacionar as citações, ou seja, 110 artigos, (2005 a 2015), dos quais todos os resumos também foram analisados para verificar a pertinência conceitual ou funcional do tema, o que possibilitou extrair qual a corrente que está em maior evidência e quais são as abordagens evolutivas.

Quanto aos procedimentos utilizados para o processamento da AFE foram seguidos a ordem recomendada na literatura: análise de KMO (acima de $0,5)$; KMO de cada item na matriz de anti imagem (acima de 0,5); exclusão de itens com comunalidade abaixo de 0,5; exclusão de itens com cargas em apenas um fator abaixo de 0,5 ; exclusão de itens com carga maior em um fator que não o original; e exclusão dos itens remanescentes de fator com confiabilidade (Alpha de Cronbach) abaixo de 0,6. Após cada depuramento, os dados foram processados novamente. A amostra é considerada adequada visto que $(\mathrm{KMO}=0,866$; Bartlett $\mathrm{p}<0,001$ ), e a variância total explicada é igual a 70,3\% (Hair et al., 2009).

Dessa forma, após cumprirem com as exigências de cada etapa de análise, foram retidas 39 obras dos autores mais referenciados, sendo agrupadas em sete fatores da matriz de componentes rotacionada, conforme pode ser visualizado no Quadro 2. 
Conceitos Clássicos e Abordagens Correntes em Inteligência Competitiva: Um Estudo Bibliométrico de 2005 a 2015

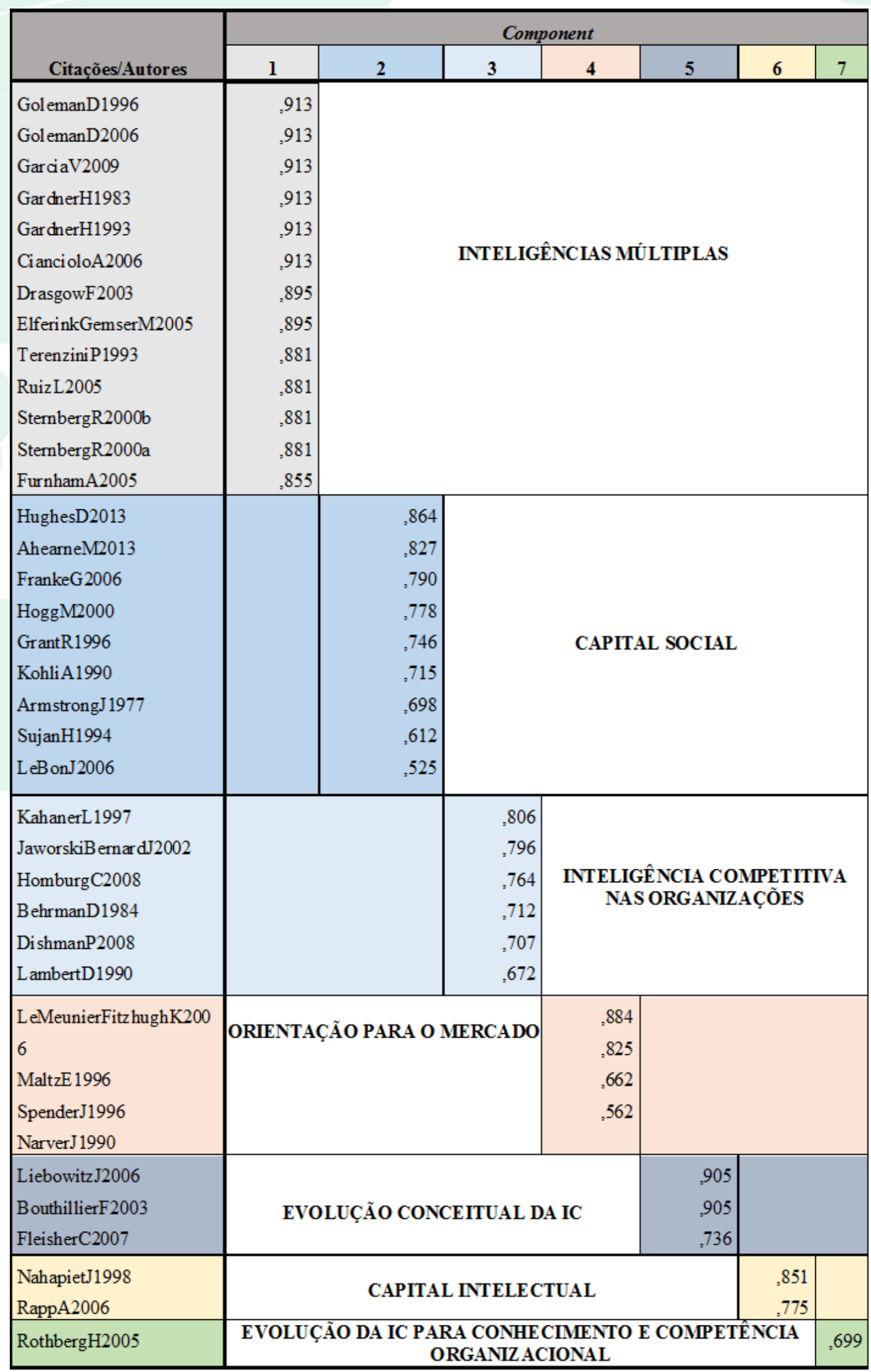

Quadro 2 - Matriz de Componentes Rotacionada

Fonte: Dados da Pesquisa, 2016 
O próximo passo foi a nomeação dos 7 fatores, ou seja, após leitura de todos os resumos das obras mais referenciadas no período (análise de citações), identificou-se os temas predominantes em seus estudos, sendo denominados de correntes conceituais ou funcionais, assim descritas:

$\checkmark \quad$ Fator 1 = Inteligências Múltiplas

$\checkmark$ Fator 2 = Capital Social

$\checkmark$ Fator $3=$ Inteligência Competitiva nas Organizações

$\checkmark$ Fator $4=$ Orientação para o Mercado

$\checkmark$ Fator $5=$ Evolução Conceitual da Inteligência Competitiva $\checkmark$ Fator $6=$ Capital Intelectual

$\checkmark$ Fator $7=$ Evolução da IC ao Conhecimento e Competência Organizacional

Com base nas correntes de cada fator, identificou-se subgrupos dentro dos temas evidenciados. Para melhor compreensão, elenca-se no Quadro 3, de forma sintetizada, os fatores, os autores, ano de publicação e as principais características das abordagens clássicas dos artigos que compõem as obras mais referenciadas na temática da Inteligência Competitiva.

\begin{tabular}{|c|c|c|}
\hline Fator & Autor/ano & Características \\
\hline \multirow[t]{5}{*}{ Inteligências Múltiplas } & $\begin{array}{l}\text { Goleman 1996; Goleman } \\
\text { 2006; Garcia 2009 }\end{array}$ & Inteligência Emocional \\
\hline & $\begin{array}{l}\text { Gardner 1983; Gardner 1993; } \\
\text { Cianciolo 2006; Drasgow } \\
\text { 2003; Sternberg 2000b; }\end{array}$ & $\begin{array}{l}\text { Inteligências Analitica, Corporal, } \\
\text { Prática Emocional, Contextual, } \\
\text { Musical, Interpessoal, Intrapessoal, } \\
\text { Ecológico, Social, Naturalista, Sexual, } \\
\text { para o Negócio, Espiritual, Espacial, } \\
\text { Matemática ou Verbal. } \\
\text { Inteligência Contextual }\end{array}$ \\
\hline & $\begin{array}{l}\text { Elferink Gemser 2005; } \\
\text { Terenzini 1993; } \\
\text { Ruiz 2005; }\end{array}$ & $\begin{array}{l}\text { Conhecimentos e Habilidades Táticas } \\
\text { Cognição }\end{array}$ \\
\hline & Sternberg $2000^{a}$ & Aplicação prática dos conhecimentos \\
\hline & FurnhamA2005 & Inteligência de negócios \\
\hline \multirow[t]{2}{*}{ Capital Social } & $\begin{array}{l}\text { Hughes 2013; Franke 2006; } \\
\text { Ahearne 2013; Sujan 1994; } \\
\text { KohliA1990; Armstrong } \\
\text { 1977; LeBon } 2006\end{array}$ & Inteligência Competitiva Interfuncional \\
\hline & Hogg 2000; Grant 1996; & Identidade Social \\
\hline $\begin{array}{l}\text { Inteligência Competitiva } \\
\text { nas Organizações }\end{array}$ & $\begin{array}{l}\text { Kahaner 1997; Jaworski } \\
\text { Bernard 2002; Homburg } \\
\text { 2008; Behrman 1984; } \\
\text { Dishman 2008; Lambert } 1990\end{array}$ & Alavancagem nos negócios \\
\hline $\begin{array}{l}\text { Orient ação para o } \\
\text { Mercado }\end{array}$ & $\begin{array}{l}\text { Le Meunier Fitzhugh 2006; } \\
\text { Maltz E1996; Narver 1990; } \\
\text { Spender 1996 }\end{array}$ & Inteligência de mercado \\
\hline $\begin{array}{l}\text { Evolução Conceitual da } \\
\text { Inteligência Competitiva }\end{array}$ & $\begin{array}{l}\text { Liebowitz 2006; Bouthillier } \\
\text { 2003; Fleisher } 2007\end{array}$ & Inteligência Estratégica \\
\hline Capital Intelectual & Nahapiet 1998; Rapp 2006 & Conhecimento \\
\hline $\begin{array}{l}\text { Evolução do } \\
\text { Conhecim ento à } \\
\text { Inteligência Competitiva }\end{array}$ & Rothberg 2005 & Vantagem competitiva \\
\hline
\end{tabular}

Quadro 3 - Análise das citações das obras seminais Fonte: Os Autores, 2016 
Houve uma concentração maior de citações e referências no fator 1 , o mesmo foi denominado de "Inteligências Múltiplas" em função da abordagem dos artigos. Outros 6 temas (vide Quadro 3) compõem os 7 subgrupos temáticos identificados. Estes tratavam do uso dos conceitos da inteligência competitiva em diferentes contextos, induzindo à inferição de que há pertinência temática em outros contextos e não apenas quando a inteligência é tratada no meio empresarial. Por exemplo, sob inteligência emocional, os aspectos cognitivos e aplicação práticas dos conhecimentos sobre inteligência, são considerados em obra de 2005, nos meios esportivos e que, de fato, referia-se à inteligência de negócios, isto é, como alavancar nos as habilidades atléticas para torná-las sustentáveis nos meios esportivos.

Surpreendeu também o segundo fator, cuja corrente diz respeito ao "Capital Social", pois as características apresentadas permeiam a preocupação com as vendas, compras, com os clientes e até mesmo com os concorrentes. $\mathrm{O}$ olhar volta-se também para a disposição social do grupo, liderança, cultura do grupo, tomada de decisão, comunicação e outros aspectos que sejam relevantes para a identidade social como algo estratégico para a empresa se diferenciar dos concorrentes.

Os conceitos da "Inteligência Competitiva Aplicada aos Negócios" são aplicados com o propósito claro e definido de alavancar os negócios. Já a "Orientação para o Mercado" aborda a inteligência de marketing propriamente dita. Destaca-se que o aspecto mercadológico envolve principalmente o marketing mix de produtos ou portfólio de produtos, envolvendo as estratégias dos 4 Ps: Produto, Preço, Ponto de Distribuição e Promoção.

Conforme mencionado no referencial deste artigo, há uma corrente pequena de pesquisa sobre a "Evolução Conceitual da Inteligência Competitiva", como pode ser visualizado no Quadro 3, somente três autores foram citados por abordar o tema de forma conceitual. Certamente essa é uma lacuna para futuras pesquisas e produções teóricas. Nota-se, em paralelo, que possivelmente a complexidade do tema (geração de teoria em inteligência competitiva), atrai menos autores.

O mesmo pode-se dizer para a corrente que defende o "Capital Intelectual", ou seja, o conhecimento criado e aplicado na organização por meio do acesso a informações externas possibilitados, via inteligência, para possível desenvolvimento de capacidades específicas na organização. Mais preocupante ainda é muito incipiente abordagem da "Evolução do Conhecimento à Inteligência Competitiva". O tema refere-se ao conhecimento e formalização dos processos de inteligência que permitem melhor manipulação de sua função na organização. As implicações são muitas, desde de domínio técnico, capacidades dinâmicas, inovação, aprendizagem organizacional, entre outras, que poderiam garantir e ampliar a vantagem competitiva. Entende-se que nesse tema cabe, em especial, estudos sobre maturidade em uso da inteligência competitiva.

Em relação à análise temática do conteúdo dos resumos dos 110 artigos internacionais, que serviram como base para a pesquisa, apresenta-se, no Quadro 4, as principais abordagens atuais da inteligência competitiva. 
Conceitos Clássicos e Abordagens Correntes em Inteligência Competitiva: Um Estudo Bibliométrico de 2005 a 2015

\begin{tabular}{|c|c|c|}
\hline Abordagem Temática Atual & Autores & Ano \\
\hline \multirow{8}{*}{ Int digências Múltiplas } & Qiu (2008); Lesca (2008) & 2008 \\
\hline & $\begin{array}{l}\text { Bers, Dismukes, Miller, \& Dubrovensky (2009); Schwarz } \\
\text { (2009) }\end{array}$ & 2009 \\
\hline & Strauss \& Toit (2010); Calof \& Smith (2010); Huggins (2010) & 2010 \\
\hline & $\begin{array}{l}\text { Garcia Alsina, Ortoll, \& López Borrull (2011); Yap \& Abdul } \\
\text { Rashid ( 2011) }\end{array}$ & 2011 \\
\hline & $\begin{array}{l}\text { Schwartz (2012); Ching Seng Yap (2012); Adidam, Banerjee, } \\
\text { \& Shukla (2012) } \\
\text { Iqbal (2012); Z. Y. Wang, Li, Li, \& Li (2012); Natalia (2012); } \\
\text { Betsy (2012); Perucchi (2012) }\end{array}$ & 2012 \\
\hline & $\begin{array}{l}\text { Ramirez, Osterman, \& Gronquist (2013); Garcia-Alsina, } \\
\text { Ortoll, \& Cobarsi-Morales (2013); Vidigal (2013) }\end{array}$ & 2013 \\
\hline & $\begin{array}{l}\text { Sewdass \& Toit (2014); Ruiz-Pérez, Graupera, \& Garcia Coll } \\
\text { (2014); Edward (2014); Kimbrough, Chou, Chen, \& Lin } \\
\text { (2014); Sewdass \& Toit (2014); Tao Jin (2014) }\end{array}$ & 2014 \\
\hline & $\begin{array}{l}\text { Safa et al. (2015); Cekuls (2015); Fernández-Pérez, } \\
\text { Garechana-Anacabe, Azkarate-Olaran, Núñez-Barrenechea, } \\
\text { \& Ortiz-de-Zárate-Garaizabal (2015) }\end{array}$ & 2015 \\
\hline \multirow[t]{8}{*}{ Capital Social } & Montgomery, Moore, \& Urbany (2005) & 2005 \\
\hline & Baars \& Kemper (2008) & 2008 \\
\hline & McMullen, Shepherd \& Patzelt (2009) & 2009 \\
\hline & González-Gálvez, Rey-Martín, \& Cavaller-Reyes (2011) & 2011 \\
\hline & Ko, Maciejewski, Jang, \& Ebert (2012) & 2012 \\
\hline & $\begin{array}{l}\text { Abrahams, Jiao, Fan, Wang, \& Zhang (2013); He, Zha, \& Li } \\
\text { (2013); Hughes, Le Bon, \& Rapp (2013); Ahearne (2013); } \\
\text { Ramirez-Calvo, P.; Triviño, A.; Berges-Garcia, A.; Meneses- } \\
\text { Chaux, J.M; Martine (2013) }\end{array}$ & 2013 \\
\hline & Mariadoss, Milewicz, Lee, \& Sahaym (2014) & 2014 \\
\hline & Rapp, Agnihotri, Baker, \& Andzulis (2015); He et al. (2015) & 2015 \\
\hline \multirow{6}{*}{$\begin{array}{l}\text { Int digência Competitiva nas } \\
\text { Organizações }\end{array}$} & Neugarten (2006) & 2006 \\
\hline & Tanev \& Bailetti (2008); Saayman et al. (2008) & 2008 \\
\hline & $\begin{array}{l}\text { Muñoz-Cañavate, Pulgarin-Guerrero, Muñoz Cañavate, \& } \\
\text { Pulgarin Guerrero (2009) }\end{array}$ & 2009 \\
\hline & Ursacesc (2010) & 2010 \\
\hline & Patter son \& Martzoukou (2012) & 2012 \\
\hline & Bagnoli \& Watts (2015); Muñoz-Cañavate \& Hipola (2015) & 2015 \\
\hline \multirow[t]{2}{*}{ Orientação para o mercado } & $\begin{array}{l}\text { Trim \& Lee (2008); Dishman \& Calof (2008); Michaeli, } \\
\text { Rainer? Simon (2008); Fleisher, Wright, \& Allard (2008) }\end{array}$ & 2008 \\
\hline & Hattula, Schmitz, Schmidt, \& Reinecke (2013) & 2013 \\
\hline \multirow{2}{*}{$\begin{array}{l}\text { Evolução } \quad \text { Conceitual } \\
\text { Int digência }\end{array}$} & Canongia (2007) & 2007 \\
\hline & Gainor \& Bouthillier (2014) & 2014 \\
\hline \multirow[t]{3}{*}{ Capital Intelectual } & $\begin{array}{l}\text { Heinrichs \& Lim (2005); Erickson \& Rothberg (2009); Yap } \\
\text { \& Abdul Rashid (2011) }\end{array}$ & 2005 \\
\hline & $\mathrm{Ma}(2012)$ & 2012 \\
\hline & Erickson, 2013 & 2013 \\
\hline \multirow{3}{*}{$\begin{array}{l}\text { Evolução da IC ao Conhecimento e } \\
\text { Competência Organizacional }\end{array}$} & Saayman et al. (2008) & 2008 \\
\hline & Heppes \& Toit (2009) & 2009 \\
\hline & Shih, Liu, \& Hsu (2010); Rogojanu (2010) & 2010 \\
\hline
\end{tabular}

Quadro 4 - Análise Temática da Abordagem Atual Fonte: Os Autores, 2016

Apesar de já inferido acima, acerca da incipiência mais evidente da abordagem dos temas sob o ponto de vista das publicações, é essa análise temática que permite identificar a tendência atual das correntes de pesquisa no âmbito da inteligência competitiva. Sob esse aspecto, "Inteligências 
Múltiplas" e "Capital Social" continuam em evidência. Da mesma forma os temas mais conceituais como "Evolução Conceitual da IC", "Capital Intelectual" e "Evolução do Conhecimento à IC" possuem pouca abordagem no cenário atual em termos de pesquisa científica.
Por outro lado, verificou-se que novas correntes foram e estão sendo pesquisadas pelos autores que escreveram no período de 2005 a 2015, além das que já eram pesquisadas, conforme exposto no Quadro 5.

\begin{tabular}{|c|c|c|}
\hline $\begin{array}{l}\text { Abordagem Temática } \\
\text { Evolutiva }\end{array}$ & Autores & Ano \\
\hline \multirow{10}{*}{$\begin{array}{l}\text { Int eligência } \\
\text { Tecnológica }\end{array}$} & Lichtenthale (2005) & 2005 \\
\hline & $\begin{array}{l}\text { Braman (2006); Lara \& Vázquez (2006); Comai, A., Tena, J., \& Vergara, } \\
\text { J.C. (2006) }\end{array}$ & 2006 \\
\hline & Jourdan, Rainer, \& Marshall (2008); Gordon (2008) & 2008 \\
\hline & Mortara (2009) & 2009 \\
\hline & de Barros \& de Souza (2010) & 2010 \\
\hline & René (2011); Yoon \& Kim (2011); Gaidelys (2011) & 2011 \\
\hline & $\begin{array}{l}\text { Zheng, Fader, \& Padmanabhan (2012); Quan, Wenyin, Dou, Xiong, \& Ge } \\
\text { (2012); Wang \& Guo (2012); Dos Santos (2012) }\end{array}$ & 2012 \\
\hline & $\begin{array}{l}\text { Fontaine (2013); Ramirez-Calvo, P.; Triviño, A.; Berges-Garcia, A.; } \\
\text { Meneses-Chaux, J.M.; Martinez (2013) }\end{array}$ & 2013 \\
\hline & $\begin{array}{l}\text { Zhang, Zhou, Porter, \& Vicente Gomila (2014); B. Wang, Liu, Ding, Liu, } \\
\text { \& Xu (2014); Luciano (2014); Ruiz-Pérez et al. (2014); Tuta (2014) }\end{array}$ & 2014 \\
\hline & Villarroel et al. (2015) & 2015 \\
\hline \multirow[t]{9}{*}{$W e b$} & Srinivasan, Menczer, \& A (2005) & 2005 \\
\hline & Vaughan, Gao, \& Kipp (2006) & 2006 \\
\hline & Bose, 2008; Vaughan \& You (2008) & 2008 \\
\hline & $\begin{array}{l}\text { Anica-Popa \& Cucui (2009); Wingyan (2009); Anica-Popa \& Cucui } \\
\text { (2009) }\end{array}$ & 2009 \\
\hline & Romero-Frias \& Vaughan (2010); Vaughan \& You (2010) & 2010 \\
\hline & Xu, Liao, Li, \& Song (2011); Del-Fresno-Garcia (2011) & 2011 \\
\hline & Yoon (2012) & 2012 \\
\hline & Ferrara, De Meo, Fiumara, \& Baumgartner (2014) & 2014 \\
\hline & Delgado (2015) & 2015 \\
\hline Capital Estrutural & $\begin{array}{l}\text { Smith (2006) } \\
\text { Zangoueinezhad \& Moshabaki (2009) }\end{array}$ & $\begin{array}{l}2006 \\
2009\end{array}$ \\
\hline \multirow{5}{*}{$\begin{array}{l}\text { Int digência } \\
\text { Competitiva nas PME }\end{array}$} & Salles (2006) & 2006 \\
\hline & Goitia, Sáenz-de-Lacuesta, \& Bilbao (2008) & 2008 \\
\hline & Molnar (2012) & 2012 \\
\hline & Kay, Youtie, \& Shapira (2014) & 2014 \\
\hline & Cantonnet, Aldasoro, \& Cilleruelo (2015) & 2015 \\
\hline
\end{tabular}

Quadro 5 - Abordagem Temática Evolutiva Fonte: Os Autores, 2016

Pode-se observar que outras 4 abordagens conceituais estão em evidência no período pesquisado. Uma delas é "Inteligência Tecnológica". O fenômeno é compreensível, porque a partir de 2003, Chesbrough popularizou a conceito de inovação aberta, que requer para sua maior eficiência, um sistema de inteligência competitiva que acesse, selecione e permita a incorporação de tecnologias externas à organização, ampliando sua base de domínio tecnológico (Rodrigues, 2012).

A segunda corrente evolutiva que mais teve publicações foi a "Web". Essa corrente reflete a preocupação das empresas em fornecer e coletar informações dos seus produtos, obter perfil de clientes e comportamentos de segmentos de mercado, por meio das redes sociais. Quanto ao "Capital Estrutural", mais entendido como sendo os investimentos feitos em sistemas de informações e à estrutura organizacional da empresa, indica que há uma preocupação em construir bases cognitivas dentro das empresas, como forma de aumentar suas capacidades e competências internas. Nesta área, é notável ainda o crescimento da inteligência nas pequenas e médias empresas. Normalmente, quando se pensa em IC pensa-se em sua 
aplicabilidade em grandes organizações. No entanto, verificou-se que há uma crescente preocupação com a utilização dos conceitos de IC também pelas Pequenas e Médias Empresas.

Infere-se que há uma lacuna maior a ser pesquisada na abordagem mais conceitual do tema, pois na maioria das vezes as pesquisas voltam-se mais para os aspectos funcionais.

\section{CONSIDERAÇÕES FINAIS}

A bibliometria realizada para esse artigo permitiu identificar os conceitos da Inteligência Competitiva conforme a abordagem dos principais autores referenciados, bem como a mensuração da evolução do tema, de forma a caracterizar as correntes conceituais e funcionais. Conclusivamente, pode-se perceber que as abordagens dos principais autores neste estudo, são mais funcionais do que conceituais.

Ao realizar o processamento estatístico dos 126 artigos internacionais pesquisados, obteve-se o agrupamento de 7 fatores, que por outro lado, representam 7 correntes de pesquisa que autores considerados seminais hoje, mais pesquisam e publicam. O primeiro fator foi denominado "Inteligências Múltiplas" pois aborda a inteligência emocional, habilidades táticas e físicas, inteligência contextual, cognição, aplicação prática dos conhecimentos. Isso não só no campo de cognição, mas também na área de negócios.

O "Capital Social" foi o segundo fator com maior carga de citações e neste os autores demonstraram uma preocupação com a inteligência competitiva interfuncional, ou seja, vendedores, compradores, concorrentes, fornecedores e clientes. Esse fator assemelhou-se bastante com o quarto fator, denominado "Orientação para o Mercado", cuja preocupação é com a inteligência de marketing. Voltase principalmente para $\mathrm{o}$ apoio às estratégias de marketing mix e determinação de perfil de clientela de segmentos de mercado.

Esperava-se um enfoque maior na terceira corrente "Inteligência Competitiva nas Organizações" da qual a teoria e os conceitos são aplicados para alavancar os negócios. Talvez pela necessidade de soluções mais pragmáticas ou talvez pelo receio de que inteligência competitiva esteja ligada à espionagem industrial, não são contributivamente significativos os conceitos produzidos ou mesmo ampliados nessa área.

Quanto às três últimas correntes que dizem respeito especificamente aos aspectos conceituais "Evolução Conceitual da IC", "Capital Intelectual" e "Evolução da IC ao Conhecimento", 6 obras apenas foram referenciadas, uma em 1998, outra em 2003, uma em 2005, duas em 2006 e outra em 2007. Quando realizada a análise temática dos resumos dos artigos compreendidos no período de 2005 a 2015, dos 110 que foram efetivamente baixados e analisados, apenas
11 abordaram as correntes conceituais, ou seja, em 10 anos foram publicados, na base de periódicos estudada, somente 11 obras que tratam dos conceitos da IC de forma evolutiva.

Da análise temática foi possível identificar ainda a evolução do tema em seu aspecto funcional cuja corrente em maior evidência é a "Inteligência Tecnológica", merecendo destaque nesta, os conceitos de Business Intelligence. A outra corrente é a "Web", indicando que as empresas estão aproveitando a mídia social para disponibilizar informação dos seus produtos e também captar informações dos seus clientes e concorrentes, visando atuar estrategicamente com as mesmas. Duas obras abordaram o "Capital Estrutural", manifestação da preocupação que as organizações estão tendo com a estrutura organizacional.

Fato interessante foram os estudos direcionados para a Inteligência Competitiva no âmbito das Pequenas e Médias Empresas, tão importante para a economia quanto para as grandes corporações e que também precisam utilizar as informações de forma estratégica para competir e manter sua vantagem competitiva.

Do estudo pode-se concluir que não existe uma única corrente conceitual sobre o tema Inteligência Competitiva, mas todas as abordagens das correntes existentes, seja no marketing, na gestão do conhecimento, nos negócios, na tecnologia e até mesmo na identidade social, permitem às organizações estruturarem-se estrategicamente para fazer frente às rápidas mudanças dos ambientes externo e interno. Entende-se que dessa forma, ao conhecer e identificar os pontos prioritários e estratégicos do negócio da empresa a mesma possa investir em inovação a fim de criar ou agregar valor ao portfólio de seus produtos, sustentando sua capacidade competitiva.

Para a teoria esse artigo contribuiu na medida em que foi possível delinear que o recurso mais valioso de uma organização é a informação, que pode ser tanto estruturada como não estruturada, utilizada de forma inteligente para a tomada de decisão. Além disso, que independe da área de origem (marketing, estratégia, negócios, TI), a informação subsidiada de forma tempestiva, acurada e confiável, torna-se fundamental, tanto para o processo decisório, quanto para a formulação de estratégias. Essa corrente permite enfatizar uma abordagem informacional inteligente, capaz de gerar conhecimento capacitativo para a organização, com todas as implicações positivas do fato.

Certamente, fica evidente a incipiência da inteligência competitiva como área do saber. Por enquanto, a inteligência parece ser vista apenas como área instrumental para gerentes e executivos. Assim, sugere-se pesquisas em todos os aspectos da inteligência, que contribuam para a sua evolução teórica. Em especial, é preciso aprofundar o estudo acerca das correntes conceituais do processo de criação da Inteligência Competitiva no âmbito dos negócios 
empresariais, bem como dispensar um enfoque maior sobre os níveis de maturidade da Inteligência Competitiva nas organizações. Faz-se mister frisar que a maior limitação desta pesquisa está no fato da utilização de apenas uma base de dados, o que nos permite sugerir a ampliação da pesquisa em outras bases.

\section{REFERÊNCIAS}

Adidam, P. T., Banerjee, M., \& Shukla, P. (2012). Competitive intelligence and firm's performance in emerging markets: An exploratory study in India. Journal of Business and Industrial Marketing, 27(3), 242-254. http://doi.org/10.1108/08858621211207252

Anica-Popa, I., \& Cucui, G. (2009). A Framework for Enhancing Competitive Intelligence Capabilities using Decision Support System based on Web Mining Techniques. International Journal of Computers, Communication \& Control, IV(4), 326334.

Baars, H., \& Kemper, H.-G. (2008). Management Support with Structured and Unstructured DataAn Integrated Business Intelligence Framework. Information Systems Management, 25(2), 132-148. http://doi.org/10.1080/10580530801941058

Bagnoli, M., \& Watts, S. G. (2015). Competitive intelligence and disclosure. RAND Journal of Economics, 46(4), 709-729. http://doi.org/10.1111/1756-2171.12103

Bers, J. A., Dismukes, J. P., Miller, L. K., \& Dubrovensky, A. (2009). Accelerated radical innovation: Theory and application. Technological Forecasting and Social Change, 76(1), 165-177. http://doi.org/10.1016/j.techfore.2008.08.013

Bose, R. (2008). Competitive intelligence process and tools for intelligence analysis. Industrial Management \& Data Systems, 108(4), 510-528. http://doi.org/10.1108/02635570810868362

Braman, S. (2006). The Micro- and Macroeconomics of Information. Annual Review of Information Science and Technology, 30, 3-52. http://doi.org/10.1002/aris.1440400108

Calof, J., \& Smith, J. (2010). The integrative domain of foresight and competitive intelligence and its impact on R\&D management. $R$ and $D$ Management, $\quad 40(1), \quad 31-39$. http://doi.org/10.1111/j.1467-9310.2009.00579.x
Canongia, C. (2007). Synergy between Competitive Intelligence (CI), Knowledge Management (KM) and Technological Foresight (TF) as a strategic model of prospecting - The use of biotechnology in the development of drugs against breast cancer. Biotechnology Advances, 25(1), 57-74. http://doi.org/10.1016/j.biotechadv.2006.10.001

Cekuls, A. (2015). Leadership Values in Transformation of Organizational Culture To Implement Competitive Intelligence Management: the Trust Building Through Organizational Culture. European Integration Studies, O(9), 244-256. http://doi.org/10.5755/j01.eis.0.9.12811

Comai, A., Tena, J., \& Vergara, J.C. (2006). Software para la vigilancia tecnológica de patentes: evaluación desde la perspectiva de los usuarios. El Profesional de La Información, 15(6), 452-458. Recuperado em 26 março, 2018, de http://doi.org/10.3145/epi.2006.nov.06

Davis, I.; Stephenson, E. (2006). Ten trends to watch in 2006. The Mckinsey Quarterly. 2006. Disponível em:

<https://www.mckinseyquarterly.com/Ten_trends_t o_watch_in_200614

de Barros, W. C., \& de Souza, C. G. (2010). Patent Documents as a Source of Technological Information in Brazil: a user study of the Program of Automatic Supply of Technological Information (PROFINT). Information Development, 26(1), 6778. http://doi.org/10.1177/0266666909358642

Del-Fresno-Garcia, M. (2011). Infosociability: Monitoring and research on the web 2.0 for decision making. Profesional De La Informacion, 20, 548-554. http://doi.org/10.3145/epi.2011.sep.09

Dishman, P. L., \& Calof, J. L. (2008). Competitive intelligence: a multiphasic precedent to marketing strategy. European Journal of Marketing, 42(7/8), 766-785.

http://doi.org/10.1108/03090560810877141

Erickson, G. S., \& Rothberg, H. N. (2009). Intellectual capital in business-to-business markets. Industrial Marketing Management, 38(2), 159-165. http://doi.org/10.1016/j.indmarman.2008.12.001

Fernández-Pérez, S., Garechana-Anacabe, G., Azkarate-Olaran, A., Núñez-Barrenechea, J., \& Ortiz-de-Zárate-Garaizabal, P. (2015). Factores influyentes, facilitadores y barreras en la implantación de una unidad de inteligencia competitiva: estudios de caso y análisis cuantitativo. El Profesional de La Información, 24(3), 310. http://doi.org/10.3145/epi.2015.may.11 
Ferrara, E., De Meo, P., Fiumara, G., \& Baumgartner, R. (2014). Web data extraction, applications and techniques: A survey. Knowledge-Based Systems, 70 , 301-323. http://doi.org/10.1016/j.knosys.2014.07.007

Fleisher, C. (2004). Competitive intelligence education: competencies, sources, and trends. Information Management Journal, 38(2), 56-63. Recuperado em 26 março, 2018, de http://www.arma.org/bookstore/files/careerpath_Fle isher.pdf

Fleisher, C. S., Wright, S., \& Allard, H. T. (2008). The role of insight teams in integrating diverse marketing information management techniques. European Journal of Marketing, 42(7/8), 836-851. http://doi.org/10.1108/03090560810877187

Fontaine, R. (2013). The evolution of pixel structures for consumer-grade image sensors. IEEE Transactions on Semiconductor Manufacturing, 26(1), http://doi.org/10.1109/TSM.2012.2237187 11-16.

Gainor, R., \& Bouthillier, F. (2014). Conceptualizing outcome and impact measures for intelligence services. Information Research, 19(1), 2016.

Garcia Alsina, M., Ortoll, E., \& López Borrull, A. (2011). Aplicaciones emergentes de inteligencia competitiva en las universidades. El Profesional de La Información, 20(5), 503-509. http://doi.org/10.3145/epi.2011.sep.03

Garcia-Alsina, M., Ortoll, E., \& Cobarsí-Morales, J. (2013). Enabler and inhibitor factors influencing competitive intelligence practices. Aslib Proceedings, 65(3), 262-288. http://doi.org/10.1108/00012531311330647

Goitia, S., Sáenz-de-Lacuesta, S., \& Bilbao, M. (2008). Implantación de sistemas de información empresarial. El Profesional de La Informacion, $17(5)$ 540-545. http://doi.org/10.3145/epi.2008.sep.08

González-Gálvez, P., Rey-Martín, C., \& CavallerReyes, V. (2011). Redes sociales para la inteligencia competitiva. Propuesta de un índice sintético. El Profesional de La Informacion, 20(5), 527-532. http://doi.org/10.3145/epi.2011.sep.06

Guedes, V. L. S., \& Borschiver, S. (2005). Bibliometria: Uma Ferramenta Estatística Para a Gestão da Informação e do Conhecimento em Sistemas de Informação, de Comunicação e de Avaliação Científica e Tecnológica. CINFORM Encontro Nacional de Ciência Da Informação, 1-
18. http://doi.org/bibliometria: uma ferramenta estatística para a gestão da informação e do conhecimento em sistemas de informação, de comunicação e de avaliação científica e tecnológica.

Guerrazzi, L. A. de C., Brandão, M. M., Campos Júnior, H., \& Lourenço, C. E. (2015). Pesquisa em Marketing e Estratégia nos Principais Periódicos Internacionais: Um Estudo Bibliométrico sobre Publicações no Século XXI. Riae - Revista IberoAmericana de Estratégia, 14. Recuperado em 26 março, 2018 de https://doi.org/10.5585/riae.v14i1.2200

Hattula, J. D., Schmitz, C., Schmidt, M., \& Reinecke, S. (2013). Is more always better? An investigation into the relationship between marketing influence and managers' market intelligence dissemination. International Journal of Research in Marketing, 32(2), 179-186.

http://doi.org/10.1016/j.ijresmar.2015.02.001

He, W., Shen, J., Tian, X., Li, Y., Akula, V., Yan, G., \& Tao, R. (2015). Gaining competitive intelligence from social media data: Evidence from two largest retail chains in the world. Industrial Management \& Data Systems, 115(9), 1622-1636. http://doi.org/10.1108/02635570710734262

He, W., Zha, S., \& Li, L. (2013). Social media competitive analysis and text mining: A case study in the pizza industry. International Journal of Information Management, 33, 464-472. Recuperado em 26 março, 2018, de http://doi.org/10.1016/j.ijinfomgt.2013.01.001

Heinrichs, J. H., \& Lim, J.-S. (2005). Model for Organizational Knowledge Creation and Strategic Use of Information. Journal of the American Society for Information Science \& Technology, 56(6), 620-629. Recuperado em 26 março, 2018, de http://doi.org/10.1002/asi.20152

Heppes, D., \& Toit, A. Du. (2009). Level of maturity of the competitive intelligence function: Case study of a retail bank in South Africa. Aslib Proceedings, $61(\mathrm{Ci})$, 48-66. http://doi.org/10.1108/00012530910932285

Hughes, D. E., Le Bon, J., \& Rapp, A. (2013). Gaining and leveraging customer-based competitive intelligence: The pivotal role of social capital and salesperson adaptive selling skills. Journal of the Academy of Marketing Science, 41(1), 91-110. http://doi.org/10.1007/s11747-012-0311-8

Jaworski, B. J. (2004). Generating Competitive Intelligence in Organizations, (Ci), 279-307. 
Jourdan, Z., Rainer, R. K., \& Marshall, T. E. (2008). Business intelligence: An analysis of the literature. Information Systems Management, 25(2), 121-131. http://doi.org/10.1080/10580530801941512

Kay, L., Youtie, J., \& Shapira, P. (2014). Signs of things to come? What patent submissions by small and medium-sized enterprises say about corporate strategies in emerging technologies. Technological Forecasting and Social Change, 85, 17-25. http://doi.org/10.1016/j.techfore.2013.09.006

Kimbrough, S. O., Chou, C., Chen, Y. T., \& Lin, H. (2014). On developing indicators with text analytics: Exploring concept vectors applied to English and Chinese texts. Information Systems and E-Business Management, 12(3), 385-415. http://doi.org/10.1007/s10257-013-0228-x

Ko, S., Maciejewski, R., Jang, Y., \& Ebert, D. S. (2012). MarketAnalyzer: An Interactive Visual Analytics System for Analyzing Competitive Advantage Using Point of Sale Data. Computer Graphics Forum, 31(3pt3), 1245-1254. http://doi.org/http://dx.doi.org/10.1111/j.14678659.2012.03117.x

Kono, C. M., Silva Jr, L. C. F. e, \& Rodrigues, L. C. (2014). Competitive intelligence, cloud computing and innovation in small enterprises. Proceedings of the 11th CONTECSI International Conference on Information Systems and Technology Management, 680-704. Recuperado em 26 março, 2018, de http://doi.org/10.5748/978859969310011 CONTECSI/PS-584

Lara, P., \& Vázquez, R. (2006). Ferroatlántica I+D y la vigilancia tecnológica. El Profesional de La Información.

Le Bon, J., \& Merunka, D. (2006). The impact of individual and managerial factors on salespeople's contribution to marketing intelligence activities. International Journal of Research in Marketing, 23(4), 395-408. Recuperado em 26 março, 2018, de http://doi.org/10.1016/j.ijresmar.2006.10.002

Ma, R. (2012). Discovering and analyzing the intellectual structure and its evolution of LIS in China, 1998-2007. Scientometrics, 93(3), 645-659. http://doi.org/10.1007/s11192-012-0702-0

Mariadoss, B. J., Milewicz, C., Lee, S., \& Sahaym, A. (2014). Salesperson competitive intelligence and performance: The role of product knowledge and sales force automation usage. Industrial Marketing Management, 43(1), 136-145. http://doi.org/10.1016/j.indmarman.2013.08.005
McMullen, J. S., Shepherd, D. A., \& Patzelt, H. (2009). Managerial (In)attention to competitive threats. Journal of Management Studies, 46(2), 157-181. http://doi.org/10.1111/j.1467-6486.2008.00799.x

Michaeli, Rainer; Simon, L. (2008). An illustration of Bayes' theorem and its use as a decision-making aid for competitive intelligence and marketing analysts. European Journal of Marketing, 42, 804 813. http://doi.org/10.1108/03090560810877169

Montgomery, D. B., Moore, M. C., \& Urbany, J. E. (2005). Reasoning About Competitive Reactions: Evidence from Executives. Marketing Science, 24(1), 138-149. http://doi.org/10.1287/mksc.1040.0076

Muñoz-Cañavate, A., \& Hípola, P. (2015). Evolution of the Online Information Industry in Spain, 19732014: actors, products and services. Online Information Review, 39(7). http://doi.org/http://dx.doi.org/10.1108/OIR-112014-0274

Muñoz-Cañavate, A., Pulgarín-Guerrero, A., Muñoz Cañavate, A., \& Pulgarín Guerrero, A. (2009). La gestión de la información en las empresas de Extremadura. Information Management by Companies in Extremadura, 32(4), 107-132. http://doi.org/10.3989/redc.2009.4.672

Neugarten, M. L. (2006). Foresight-Are we looking in the right direction? Futures, 38(8), 894-907. http://doi.org/10.1016/j.futures.2005.12.013

Patterson, L., \& Martzoukou, K. (2012). An examination of Canadian information professionals' involvement in the provision of business information synthesis and analysis services. Journal of Librarianship and Information Science, 44(1), 47-64. http://doi.org/10.1177/0961000611426215

Qiu, T. (2008). Scanning for competitive intelligence: a managerial perspective. European Journal of Marketing, 42, 814-835. http://doi.org/10.1108/03090560810877178

Quan, X., Wenyin, L., Dou, W., Xiong, H., \& Ge, Y. (2012). Link graph analysis for business site selection. Computer, 45(3), 64-69. http://doi.org/10.1109/MC.2011.260

Ramirez, R., Osterman, R., \& Gronquist, D. (2013). Scenarios and early warnings as dynamic capabilities to frame managerial attention. Technological Forecasting and Social Change, 80(4), 825-838. http://doi.org/10.1016/j.techfore.2012.10.029 

2005 a 2015

Ramirez-Calvo, P.; Triviño, A.; Berges-Garcia, A.; Meneses-Chaux, J.M.; Martínez, J. F. (2013). Nuevas Tecnologías en Análisis de Inteligencia Competitiva. Casos Prácticos. El Profesional De La Información, 448-454. Retrieved from http://nebulosa.icesi.edu.co:2516/ehost/pdfviewer/p dfviewer?vid=3\&sid=4e66e457-29fc-4a64-bd555748281791a3@sessionmgr4002\&hid=4204

Rapp, A., Agnihotri, R., Baker, T. L., \& Andzulis, J. M. (2015). Competitive intelligence collection and use by sales and service representatives: how managers' recognition and autonomy moderate individual performance. Journal of the Academy of Marketing Science, 43(3), 357-374. http://doi.org/10.1007/s11747-014-0384-7

Rodrigues, L. C. Technical Competitive Intelligence System: An Innovation and Technology Management Tool. In: Nejdet Delener (ed.) Service Science Research, Strategy, and Innovation: Dynamic Knowledge Management Methods, p. 207-232. Hershey (PA): IGI Global, 2012. Premier Reference Book.

Rodrigues, L. C., Rechziegel, W., Esteves, G., \& Pereira Fernandes, M. (2012). Competitive Intelligence As Business Process Innovation. Review of Administration and Innovation - RAI, 9(4). Recuperado em 26 março, 2018, de http://doi.org/10.5773/rai.v9i4.1113

Rodrigues, L. C., \& Riccardi, R. (2007). Inteligência competitiva nos negócios e organizações. MaringáPR.

Romero-Frías, E., \& Vaughan, L. (2010). Patterns of web linking to heterogeneous groups of companies: The case of stock exchange indexes. Aslib Proceedings: New Information Perspectives, 62(2), 144-164.

http://doi.org/10.1108/00012531011034964

Ruiz-Pérez, L. M., Graupera, J. L., \& Garcia Coll, V. (2014). Inteligencia contextual percibida en el deporte. Desarrollo y validación de un cuestionario. Cultura, Ciencia Y Deporte, 9, 211-224. http://doi.org/10.2466/pms.1985.60.3.943

Saayman, A., Pienaar, J., Pelsmacker, P. de, Viviers, W., Cuyvers, L., Muller, M.-L., \& Jegers, M. (2008). Competitive intelligence: construct exploration, validation and equivalence. Aslib Proceedings (Vol. 60). Recuperado em 26 março, 2018, http://doi.org/10.1108/00012530810888006

Safa, M., Shahi, A., Haas, C. T., Fiander-McCann, D., Safa, M., Hipel, K., \& MacGillivray, S. (2015). Competitive intelligence (CI) for evaluation of $\begin{array}{lccr}\text { construction } & \text { contractors. } & \text { Automation in } \\ \text { Construction, } & 59, & 149-157 . \\ \text { http://doi.org/10.1016/j.autcon.2015.02.009 }\end{array}$

Schwartz, M. S. (2012). The State of Business Ethics in Israel: A Light Unto the Nations? Journal of Business Ethics, 105(4), 429-446. http://doi.org/10.1007/s10551-011-0975-x

Sewdass, N., \& Toit, A. Du. (2014). Current state of competitive intelligence in South Africa. International Journal of Information Management, 34(2), $185-190$. http://doi.org/10.1016/j.ijinfomgt.2013.10.006

Shih, M. J., Liu, D. R., \& Hsu, M. L. (2010). Discovering competitive intelligence by mining changes in patent trends. Expert Systems with Applications, 37(4), 2882-2890. http://doi.org/10.1016/j.eswa.2009.09.001

Sierra, J., \& Rodrigues, L. (2011). Maturidade Organizacional em Inteligência Competitiva - O Caso de uma Instituição Financeira Brasileira. XXXV Encontro Da ANPAD, 1-15.

Srinivasan, P., Menczer, F., \& A, G. P. (2004). General Evaluation Framework for Topical Web Crawlers. In Information Retrieval, Submitted, Accessed May, 417-447.

Strauss, a. C., \& Toit, a. S. a. Du. (2010). Competitive intelligence skills needed to enhance South Africa's competitiveness. Aslib Proceedings, 62(3), 302320. http://doi.org/10.1108/00012531011046925

Tanev, S., \& Bailetti, T. (2008). Competitive intelligence information and innovation in small Canadian firms. European Journal of Marketing, 42(7/8), 786-803. http://doi.org/10.1108/03090560810877150

Trim, P. R. J., \& Lee, Y. I. (2008). A strategic marketing intelligence and multi-organisational resilience framework. European Journal of Marketing, 42(7/8), 731-745. http://doi.org/10.1108/03090560810877123

Vaughan, L., Gao, Y., \& Kipp, M. (2006). Why are hyperlinks to business Websites created? A content analysis. Scientometrics, 67(2), 291-300. http://doi.org/10.1007/s11192-006-0100-6

Vaughan, L., \& You, J. (2008). Content assisted web co-link analysis for competitive intelligence. Scientometrics, 77(3), 433-444. http://doi.org/10.1007/s11192-007-1999-y 
Vaughan, L., \& You, J. (2010). Word co-occurrences on Webpages as a measure of the relatedness of organizations: A new Webometrics concept. Journal of Informetrics, 4(4), 483-491. http://doi.org/10.1016/j.joi.2010.04.005

Villarroel, G., Fernández, O., Arriagada, V., Implementación, D. E., Unidad, D. E. U. N. A., Tecnológica, D. E. V., ... Catalina, O. (2015). Diseño E Implementacion.

Wang, B., \& Guo, X. (2012). Online recruitment information as an indicator to appraise enterprise performance. Online Information Review, 36(6), 903-918.

http://doi.org/10.1108/14684521211287954

Wang, B., Liu, S., Ding, K., Liu, Z., \& Xu, J. (2014). Identifying technological topics and institutiontopic distribution probability for patent competitive intelligence analysis: a case study in LTE technology. Scientometrics, 101(1), 685-704. http://doi.org/10.1007/s11192-014-1342-3

Wang, Z. Y., Li, G., Li, C. Y., \& Li, A. (2012). Research on the semantic-based co-word analysis. Scientometrics, $\quad$ 90(3), 855-875. http://doi.org/10.1007/s11192-011-0563-y

Xu, K., Liao, S. S., Li, J., \& Song, Y. (2011). Mining comparative opinions from customer reviews for Competitive Intelligence. Decision Support Systems, 50(4), 743-754. Recuperado em 26 março, 2018, de http://doi.org/10.1016/j.dss.2010.08.021

Yap, C. S., \& Abdul Rashid, M. Z. (2011). Acquisition and strategic use of competitive intelligence. Malaysian Journal of Library and Information
Science, 16(1), 125-136.

Yoon, J. (2012). Detecting weak signals for long-term business opportunities using text mining of

Web news. Expert Systems with Applications, 39(16), 12543-12550.

http://doi.org/10.1016/j.eswa.2012.04.059

Yoon, J., \& Kim, K. (2011). Identifying rapidly evolving technological trends for R\&D planning using SAO-based semantic patent networks. Scientometrics, $\quad 88(1), \quad 213-228$. http://doi.org/10.1007/s11192-011-0383-0

Zangoueinezhad, A., \& Moshabaki, A. (2009). The role of structural capital on competitive intelligence. Industrial Management \& Data Systems, 109(2), 262-280.

http://doi.org/10.1108/02635570910930136

Zhang, Y., Zhou, X., Porter, A. L., \& Vicente Gomila, J. M. (2014). How to combine term clumping and technology roadmapping for newly emerging science \& technology competitive intelligence: "problem \& solution" pattern based semantic TRIZ tool and case study. Scientometrics, 1-15. http://doi.org/10.1007/s11192-014-1262-2

Zheng, Z. E., Fader, P., \& Padmanabhan, B. (2012). From Business Intelligence to Competitive Intelligence: Inferring Competitive Measures Using Augmented Site- Centric Data From Business Intelligence to Competitive Intelligence: Inferring Competitive Measures Using Augmented Site-Centric Data. Information Systems Research Publication, 23(3), 698-720. http://doi.org/10.1287/isre.1110.0385 